\title{
High-Energy Collisional Activation Studied via Angle-Resolved Translational- Energy Spectra of Survivor Ions*
}

\author{
P. Thibault, A. J. Alexander, ${ }^{\dagger}$ and R. K. Boyd \\ Institute for Marine Biosciences, National Research Council, Halifax, Nova Scotia, Canada
}

\begin{abstract}
Angle-resolved translational energy spectroscopy has been applied to $\mathrm{Cs}_{4} \mathrm{I}_{3}^{+}$ions that survived $8 \mathrm{keV}$ collisions with a range of collision gas targets, including inert gases and deuterium. The experimental data comprise values of the translational energy loss $\Delta T_{R}$ as a function of the (laboratory-frame) scattering angle $\theta_{\mathrm{R}}$ for each collision gas under conditions such that single-collision events dominated the scattering. The values of $\Delta T_{R}$ increase with $\theta_{R^{r}}$ in accordance with very general expectations. However, for any value of $\theta_{R}$, the values of $\Delta T_{R}$ for helium and deuterium as targets were almost indistinguishable from one another but were at least five to six times larger than those for neon and all other collision gases. These data have been shown to be consistent with theoretical considerations based upon conservation of energy and linear momentum. Theoretical approaches include the simple "elasticlimit" model, which makes no mechanistic assumptions, and a particular "binary-model" theory, which excludes electronic excitation as a possibility. Both theories are consistent with the experimental data and interpret the surprisingly large values of $\Delta \mathrm{T}_{R}$ for low-mass targets in terms of large recoil energies of the target required to ensure conservation of momentum. The most likely alternative candidate as sink for $\Delta T_{R}$ is internal excitation of the target, but this possibility was excluded in the present work by choosing $\Delta \mathrm{T}_{\mathrm{R}}$ values less than the lowest excitation energies of the inert gas targets. Moreover, such an interpretation cannot explain the similar results obtained using helium and deuterium, which were markedly different from those obtained for all other collision gases. (7 Am Soc Mass Spectrom 1993, 4, 835-844)
\end{abstract}

$\mathrm{T}$ The phenomenon of collision-induced dissociation (CID) of ions has been of practical and theoretical interest for some time. In particular, CID arising from collisions between reactant ions and thermal target gas atoms (or molecules), at energies in the kiloelectronvolt range, has been characterized as proceeding via two well-separated steps, that is, a fast collisional-activation (CA) step followed by the dissociation itself on a slower time scale. Although this distinction between the $\mathrm{CA}$ step and the overall $\mathrm{CD}$ process is not universally made, it is importànt for the present work in which the two steps are separated experimentally.

Seminal work on understanding the CA processes involved in CID of small ions (few atoms only) was due to Durup [1]; this work was updated and extended by Los and Govers [2], with particular reference to diatomic reactant ions. Subsequent work on the

\footnotetext{
NNRCC No. 34881

Current address: Bristol-Myers-Squibb Pharmaceutical Research Institute, P.O. Box 4755, Syracuse, NY 13221.

Address reprint requests to Robert $\mathrm{K}$. Boyd, Institute for Marine Biosciences, National Research Council, 1411 Oxford Street, Halifax, Nova Scotia, B3H 3Z1, Canada.
}

CA process at kiloelectronvolt energies has attempted to apply to larger ions the understanding achieved by these fundamental studies [1, 2]. The elegant work of Futrell and co-workers [3-5] is notable in this respect and has extended the earlier fundamental work on diatomic ions to systems of chemical interest. Parallel with these physical chenuistry studies, attempts have been made to relate the principles thus established to CID experiments using analytical mass spectrometers. For example, the interpretation of the data [6] on variation of kinetic energy release, in CID of organic ions $(<200 \mathrm{Da})$ with scattering angle, relied upon the insights from earlier CID work [1,2]. Recent work by Cooks and co-workers [7-9], on ions of similar size, has further extended the understanding of CID of ions of chemical interest. An excellent review of current understanding of the $C A$ process has been published by McLuckey [10].

Among the studies of the CA process in analytical mass spectrometers, certain observations concerning the CID of larger organic ions have been of practical and theoretical interest [11-17]. These observations, initially published by Derrick and co-workers [11, 12, $15,16]$, concern the CID of large organic reactant ions as studied by mass-analyzed ion kinetic energy spec- 
trometry (MKKES). The fragment ions appeared at values of the electric sector field $\mathbf{E}$ much lower than those predicted by eq 1 , which assumes negligible fractional transformation of translational to internal energy in the $\mathrm{CA}$ step and also ignores any translational energy release to the products of the dissociation:

$$
E_{P}=E_{R} \times\left(m_{\Gamma} / m_{R}\right)
$$

where subscripts $R$ and $P$ denote reactant and product ions, respectively, and $\mathrm{E}$ (bold type) denotes the electric sector field strength as distinct from the symbol $\mathrm{E}$, which denotes the physical object (electric sector) itself. Large discrepancies between experimental values of $E_{\Gamma}$ and those predicted by eq 1 imply correspondingly large losses of translational energy [11-17] in the CA process.

Of course, the basic concept of the CA method involves increasing the internal energy $\epsilon_{R}$ at the expense of translational energy $T_{R}$. so that some discrepancy between predicted and observed $E_{P}$ values must in principle exist. It has been the surprisingly large magnitude of the translational energy losses $\Delta T_{R}$ of the reactant ions, up to $50 \mathrm{eV}$ and even higher, that has aroused interest [11-17]. The most obvious interpretation of such observations involves an assumption that most of $\Delta T_{R}$ is transformed into $\epsilon_{R}$ to meet large requirements of internal energy (kinetic shift) over and above the dissoctation threshold. Such kinetic shifts are required to permit the dissociation rate constants $\mathrm{k}_{d}$ to rise to values (of the order of $10^{6} \mathrm{~s}^{-1}$ ) that match the expetimental time scale, which in turn is determined solely by the physical dimensions of the apparatus used and by the speed with which the ions traverse it. For larger organic ions (frequently protonated peptides [11-17]), the large number of effective vibrational modes demands a correspondingly large value of $\epsilon_{R}$ in order that $k_{d}$ can attain values demanded by the observational time window. These large values of $\epsilon_{R}$ can be provided only by conversion of $T_{R}$ by the $C A$ step or from precollision internal energy $\epsilon_{\mathrm{R}}^{o}$ deposited in the reactant ions by the ionization process itself.

Although this kinetic-shift effect must indeed be operative, it appears that it cannot on its own account for all of the relevant experimental observations. For example, the values of $\Delta \mathrm{T}_{R}$ (measured as $\Delta \mathrm{T}_{\mathrm{P}}$ and converted via eq 1 ) observed in all cases studied [11-17] are much larger for light collision targets $\mathrm{CH}_{2}$, $\mathrm{D}_{2}, \mathrm{He}$ ) than for all others $\left(\mathrm{Ne}_{f} \mathrm{Ar}, \mathrm{Kr}, \mathrm{Xe}, \mathrm{N}_{2}\right)$ in the same apparatus. This observation can be rationalized in terms of $\Delta \epsilon_{\mathrm{K}}$ (kinetic shift), as the predominant sink for $\Delta T_{R}$, only if $C A$ by the light targets yields a significant fraction of activated ions in an isolated state which cannot undergo the dissociation reactions monitored. However, $\mathrm{Cs}_{n+1} \mathrm{I}_{n}^{+}$ions, of masses comparable to those of the large organic ions of interest but with fewer atoms (e.g., 21 vibrational modes for $\mathrm{Cs}_{5} \mathbf{I}_{4}^{+}$compared with approximately 500 for a peptide ion of similar mass) and presumably very different electronic states, display comparabie losses of translational energy $\Delta T_{R}$ in CID-MIKFS experiments. Finally, the peak shapes observed in CID-MIKES experiments are also strongly dependent on the nature of the target gas. Highly asymmetric peaks, with extensive tailing on the low-energy side, are observed for $\mathrm{He}, \mathrm{H}_{2}$, and $\mathrm{D}_{2}$, while much more narrow and symmetric peaks are observed when other target gases are used [17].

Two different approaches have been proposed to account for the surprisingly large values of $\Delta T_{R}$, and for their dependence upon the nature of the target gas. That suggested by Bricker and Russell [13] proposes that those CA. collisions that lead to observable CID products are necessarily accompanied by excitation (possibly including ionization) of the target. On the other hand, Derrick and co-workers $[11,12,15,16]$ and ourselves [17] have accounted for the experimental observations on the assumption that recoil translational energy of the target is a major sink for $\Delta T_{R}$.

The present work has attempted to clarify this problem by making observations on reactant ions that suryived the CA process sufficiently long ( $25 \mu \mathrm{s}$ or longer) so that their translational energies could be measured. In the companion article, the delayed dissociation spectra of these survivor ions, selected via their $\Delta T_{R}$ values, are used to estimate their internal energies. This approach represents an extension of earlier work by Cerny and co-workers [18, 19], who studied the low-energy tails on MIKES peaks corresponding to reactant ions that survived the CA process; preliminary accounts of these delayed dissociation studies have been published $[20,21]$. (It is worthwhile to note that the lowenergy tails of interest here [18-21] differ from those studied by Ballard and Gaskell [22], which apply to MIKE spectra for both CID and spontaneous dissociations of reactant ions formed by fast-atom bombardment only, and concerns background signals as low as $0.5 \times \mathrm{E}_{\mathrm{R}}$.)

This article reports measurements of translational energies of reactant ions that survived $\mathrm{CA}$ as a function of scattering angle. Previous investigations, using the MIKES technique, of the angular dependence of kiloelectronvolt collisions without immediate chemical consequences have involved electronic excitation and ionization (charge stripping) of simple monatomic and diatomic reactant ions [23]. This work demonstrated the necessity for ion-beam collimation both before and after the collision in order to cleanly separate the collisional deflection from the energy dispersion by the electric sector, and thus adequately specify the angle. Because such collimation dramatically reduces the intensity of the reactant ion beam, it was necessary to choose a reactant ion available at high yield, and the $\mathrm{Cs}_{4} \mathrm{I}_{3}^{\prime}$ ion fulfilled this requirement. In addition, there is no possibility that this reactant ion will produce fragment tons via losses of a few Da (e.g., lasses of hydrogen atoms), thus avoiding ambiguities in the identification of ions observed at a few electronvolts below the unscattered reactant peak in the MIKE spec- 
trum. Finally, since the $\mathrm{Cs}_{4} \mathrm{I}_{3}^{+}$ions were produced by bombardment of a "dry" CsI sample, the problems associated with collisional desolvation of ions sputtered from liquid matrices [22] were avoided.

Simple considerations of energy conservation for the $\mathrm{CA}$ step yield the expression given in eq 2 for the translational energy loss $\Delta T_{R}\left(=T_{R}^{O}-T_{R}\right)$ :

$$
\begin{aligned}
\Delta T_{R} & =\left[\left(\epsilon_{R}-\epsilon_{R}^{o}\right)+\left(\epsilon_{G}-\epsilon_{G}^{\circ}\right)+h \nu\right] \\
+\Delta T_{G} & =Q+\Delta T_{G}
\end{aligned}
$$

where superscript ${ }^{\circ}$ denotes a precollision value, $\Delta \mathrm{T}_{\mathrm{G}}$ $\left(=T_{G}-T_{G}^{o}\right)$ the translational energy gained by $G$ in the collision, and $Q$ the total inelasticity. Little is known about the radiation $h \nu$ emitted by larger ions of chemical interest during or immediately following kiloelectronvolt collisions. Experiments on extremely low yields of UV-visible emission from small ions, in collision with gases or electrons, have been reported [24-28]. It has been suggested [27, 28] that most of this UV radiation may originate from excited neutral molecules formed by electron transfer from the target gas. In cases where this electron transfer is exothermic, it is possible to observe radiation from the target gas also. However, it must be emphasized that the intensity of this radiation is generally extremely low (close to the limits of detection [29]), consistent with earlier considerations $[25,30]$ of a low probability that emission in this frequency range can compete with internal conversion to the electronic ground state in all but a small number of exceptional cases. Infrared emission from vibrationally excited ions has been studied by Dunbar and co-workers [30-33] and shown to occur on a time scale of several seconds. Further, in the present context, the fact that the energy discrepancies of interest (several electronvolts) are comparable for reactant ions, which are very different chemically but have similar masses (e.g., $\mathrm{Cs}_{\mathrm{n}+1} \mathrm{I}_{\mathrm{n}}^{+}$and protonated peptides), makes it highly unlikely that the large $\Delta \mathrm{T}_{R}$ values could be accounted for in terms of emitted photons. For all these reasons, it will be assumed here that radiation does not play a significant role in the energy balance expressed in eq 2 .

By choosing inert gas targets $\mathrm{G}$, the values of $\left(\epsilon_{\mathrm{G}}-\right.$ $\epsilon_{\mathrm{G}}^{\circ}$ ) become the well-known excitation energies of these atomic species, so that target excitation [13] can be removed from consideration in the present work by restricting experimental values of $\Delta T_{R}$ to be less than the appropriate first excitation energies (Table 1). (A few experiments involving diatomic targets will be reported here for purposes of comparison.) All quantities in eq 2, other than $\epsilon_{\mathrm{G}}$ for inert gas targets $\mathrm{G}$, are to be understood as most-probable values representative of appropriate distributions.

\section{Experimental}

Experiments were conducted using a VG Analytical $\mathrm{ZAB}-\mathrm{EQ}$ tandem hybrid instrument (BEqQ configura-
Table 1. Lowest excitation energies $\left({ }^{2} \mathrm{P}_{3 / 2}^{\mathrm{O}}\right.$ for all except helium, ${ }^{3} \mathrm{~S}_{1}$ ), and ionization energies, for the inert gases

\begin{tabular}{ccc}
\hline Gas & $\begin{array}{c}\text { Energy (eV), 1st } \\
\text { excited state }\end{array}$ & Ianization energy (eV) \\
\hline \hline $\mathrm{D}_{\mathrm{z}}$ & 11.37 & 15.43 \\
$\mathrm{He}$ & 19.819 & 24.580 \\
$\mathrm{Ne}$ & 16.619 & 21.559 \\
$\mathrm{Ar}$ & 11.548 & 15.755 \\
$\mathrm{Kr}$ & $\mathbf{9 . 9 1 5}$ & 13.996 \\
$\mathrm{Xe}$ & $\mathbf{8 . 3 1 5}$ & 12.127 \\
\hline
\end{tabular}

From C. E. Moore, Atomio Energr Levels as Derived from the Analyses of Optical Spectra, U.S. National Bureau of Standards. NSADS-NBS 35, 1971. Values for $\mathrm{D}_{2}$ are from $\mathrm{G}$. Herzberg, Spectra of Diatomic Molecules, Van Nostrand, New York, 1950.

tion), but in the experiments reported here, the tandem quadrupole assembly was not employed. The CsI sample was used as a dry powder, deposited on the probe in solution; the primary beam was $30 \mathrm{keV} \mathrm{Cs}{ }^{+}$. In collision experiments, the collision gas was used at as low a pressure as possible, consistent with adequate signal/noise ratios in the resulting spectra. These pressures were such that reactant beam intensities were attenuated by about 15-20\%. Using the approach of Kim [34], it was estimated that under these experimental conditions, approximately $90 \%$ of the attenuation was due to single-collision events. Experiments using helium, with attenuations in the range $15-85 \%$, showed no significant variation in $\Delta \mathrm{T}_{\mathrm{R}}$ versus $\theta_{\mathrm{R}}$ (see below) up to $50 \%$ attenuation.

The BEqQ instrument used here has all the same features as those described previously [35] for a related instrument but, in addition, has a high-field magnet with extended mass-range optics and a VG 11-250 datasystem for instrument control and data acquisition. In particular, facilities for beam collimation and angular selection, similar to those described previously $[23,35]$, were added to the standard ZAB-EQ instrument. Prior to the collision cell, z-collimation was achieved using the standard z-restrictor between ion source and magnet, plus an additional special z-restrictor at the point of intermediate focus between $\mathbf{B}$ and $\mathbf{E}$; $y$-collimation was achieved by a combination of the variable $\alpha$-slit, located between source and $B$, and a special extra slit located about $10 \mathrm{~cm}$ before the slit at the intermediate focal point between $B$ and $E$. In this way, observations were restricted as far as possible to events occurring in the $\mathrm{xz}$ plane via $\mathrm{y}$-collimation. (The main optical axis is defined here as the $x$-axis, so the focusing and dispersion occur in the xy-plane.) The angular dependence of the scattered intensity was investigated by using a $1-\mathrm{mm}$-high aperture that could be moved in the z-direction (slit-height direction) at the double-focus point (image slit of the electric sector E). At its maximum displacement of 5 mun from the central axis, this aperture lay on a linear trajectory, originating at the collision cell, at an angle of $0.29 \pm$ $0.03^{\circ}$ to the main axis ( $x$-axis). This method of defining 
scattering angles has been used previously $[6,17,23]$ on instruments similar to that used here. Use of an electrically floated collision cell, to more narrowly specify the location of the scattering events and thus the magnitude of the laboratory-frame scattering angle $\theta_{\mathrm{R}}$, is applicable [17] to fragment ions but not to reactants.

\section{Results and Discussion}

\section{Experimental Investigations}

In these experiments, for which the beam collimation and angular selection facilities of the instrument were used, the reactant ion beam was collimated to the maximum possible extent consistent with adequate signal/noise ratios. The ideal condition [23], in which observations are restricted to scattering events occurring in the $\mathrm{xz}$-plane, could not be satisfied fully, as discussed below.

The translational energy spectra shown in Figures 1 and 2 for $\mathrm{Cs}_{4} \mathrm{I}_{3}^{+}$reactant ions in collision with helium and neon, respectively, were obtained using an effec-

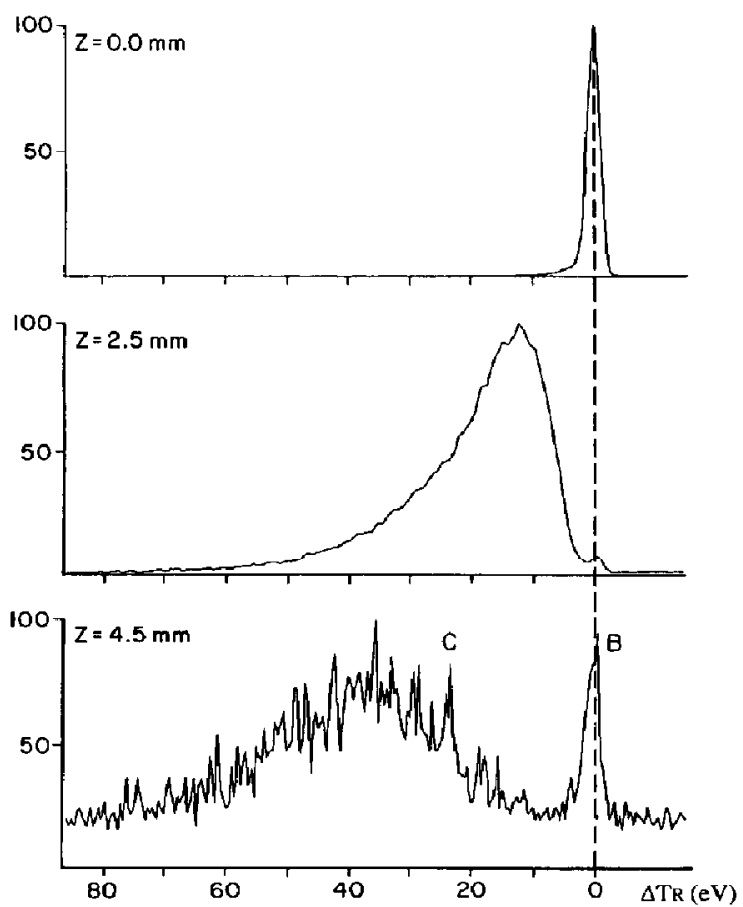

Figure 1. Translational energy spectra of $\mathrm{Cs}_{4} \mathrm{I}_{3}^{+}$reactant ions ( $m / z$ 912) after collision with helium, at different displacements of a 1-mm moveable z-aperture; a $1-\mathrm{mm}$ displacement corresponds to an angle with the principal optical axis of $0.06^{\circ}$ at the collision cell, so the z-displacements correspond to angles of $0.00 \pm 0.03^{\circ}, 0.14 \pm 0.03^{\circ}$, and $0.26 \pm 0.03^{\circ}$. Initial translational energy was $7936.0 \mathrm{eV}$, with a full-beam width at half height of 3 eV. Peak B corresponds to ions of types (1) and (2) (see text, unscattered and elastically scattered ions), while Peak C corre sponds to inelastically scattered ions (type (3))

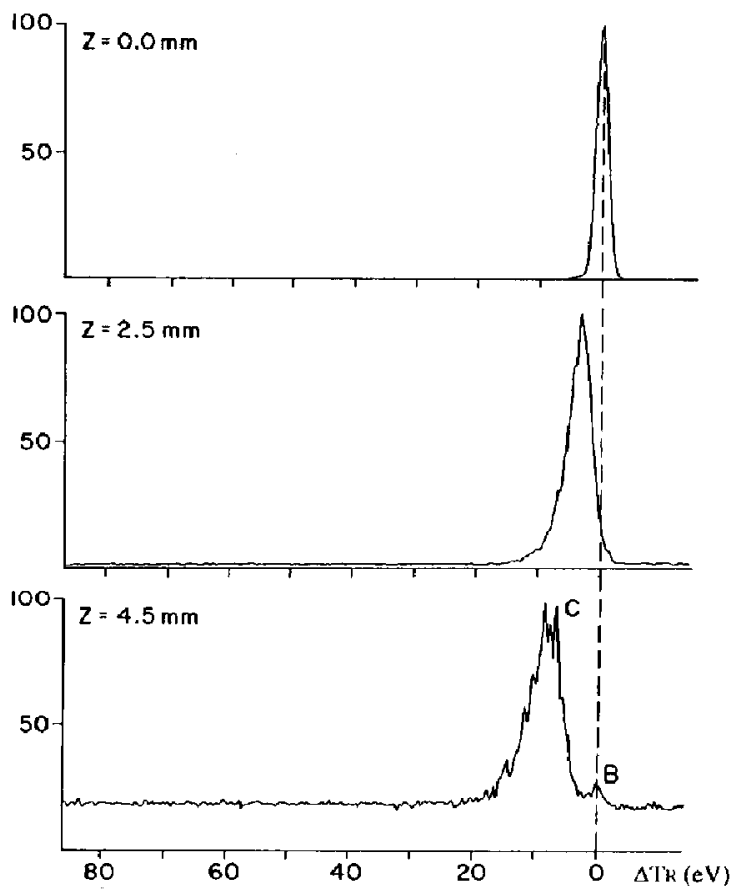

Figure 2. Iranslational energy spectra of $\mathrm{Cs}_{4} \mathrm{I}_{3}^{+}$reactant ions ( $m / z$ 912) after collision with neon, at different displacements of a 1-mm moveable z-aperture; a 1- $\mathrm{mm}$ displacement corresponds to an angle with the principal optical axis of $0.06^{\circ}$ at the collision cell, so the z-displacements correspond to angles of $0.00 \pm 0.03^{\circ}, 0.14 \pm 0.03^{\circ}$, and $0.26 \pm 0.03^{\circ}$. Initial translational energy was $7936.0 \mathrm{eV}$, with a full-beam width at half height of 3 eV. Peak B corresponds to ions of types (1) and (2) (see text, unscattered and elastically scattered ions), while Peak C corresponds to inelastically scattered ions (type (3)).

tive angular resolution of approximately $\pm 0.03^{\circ}$. Similar results were obtained for several collision gases, and the results are summarized in Figure 3. Note the similarity between the results for $\mathrm{He}$ and $\mathrm{D}_{2}$ (essentially indistinguishable from one another, although the values of $\Delta T_{R}$ for He are consistently slightly larger than for $\mathrm{D}_{2}$ ), and the marked separation between these results and those for all other collision gases. The important property of the collision target in these experiments, therefore, appears to be its mass, or possibly some other property closely correlated with mass; excitation and ionization energies (Table 1) do not correlate well with the observations summarized in Figures 1-3. It is true that one of the crucial comparisons ( $\mathrm{He}$ with $\mathrm{D}_{2}$ ) involves a monatomic and a diatomic target, but no other option is available for this particular comparison.

The trends evident in Figures $1-3$ can be understood qualitatively in terms of three kinds of ions arriving at the slit at the double-focus point:

1. Ions that have undergone no collisions whatsoever;

2. Ions that have experienced elastic collisions $(Q=$ $0)$; and 


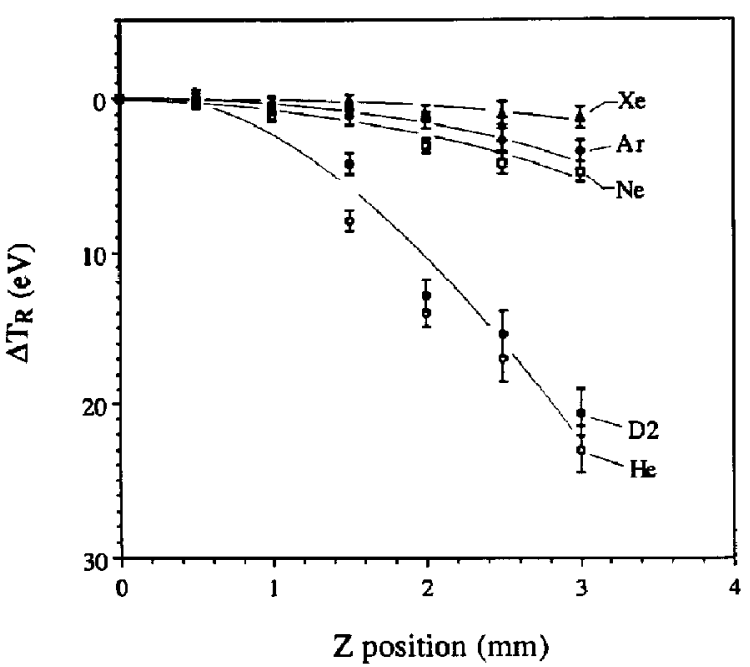

Figure 3. Must probable values of translational energy loss $\Delta T_{R}=\left(T_{R}^{\circ}-T_{R}\right)$ for $\mathrm{Cs}_{4} \mathrm{I}_{3}^{+}$ions, for different collision targets, as a function of scattering angle (inelastically scattered ions, type (3)). A displacement of the movable z-aperture by $1 \mathrm{~mm}$ corresponds to an angle of $0.06 \pm 0.03^{\circ}$ at the collision cell. Experimental uncertainties for $D_{2}$ and for $\mathrm{He}$ are standard deviations for pooled experiments conducted on two occasions.

3. Ions that have undergone inelastic collisions $(Q>$ 0 ) involving significant internuclear momentum transfer (and thus necessarily involving rotational/vibrational excitation, but not necessarily excluding simultaneous electronic excitation [1]).

Ions in categories 1 and 2 are expected to dominate the translational energy spectra at low scattering angles, and to correspond to values of $\Delta \mathrm{T}_{\mathrm{R}}$ that are zero (type 1) or small (type 2). Those ions falling into category 3 , however, should become of greater relative intensities, and show increasing values of $\Delta T_{R}$, at larger angles. These predictions are borne out by Figures 1 and 2, in which the ions of types 1 plus 2 (peak $B$ ) have been successfully resolved from those of type 3 (peak C). The survival of peak $B$ at the larger z-displacements, evident in some of these spectra, reflects residual cross talk between energy dispersion and angular selection; restriction (via beam collimation) to events in the $x z$-plane has been shown [23] to be a necessary condition for complete elimination of such cross talk in apparatus of the kind used here. This condition could not be strictly fulfilled in the present work because of beam intensity limitations.

Another striking trend evident from Figures 1-3 concerns the much larger values of $\Delta T_{R}$, at a given z-displacement (scattering angle $\theta_{\mathrm{R}}$ ), observed for helium compared with those for neon and all heavier target gases. This trend with target mass is also evident in CID-MIKES experiments [11-17] and in previous experiments of the present kind $[18,19]$ but without angular selection, that is, integrated over the full instrumental range for $z$ (and thus for $\theta_{R}$ ). A similar dependence on target mass was also noted [36] in measurements of excess translational energy of ionized target atoms produced by $4 \mathrm{keV}$ collisions with neutral projectiles, as discussed further below.

\section{Theoretical Considerations}

The present experimental investigations of angular dependence of $\mathrm{CA}$ events demand a theoretical treatment that includes scattering via momentum transfer. The present work is thus very different from that described by Los and Govers [2] in which the nature of excitedstate potential curves of diatomic reactant ions was deduced from translational energy spectra of the product ions, using the explicit assumption that the $\mathrm{CA}$ step involves only long-range electronic excitation with negligible internuclear momentum transfer. Two versions of the momentum-transfer model of CA were used in the present work.

The elastic-limit model does not pretend to calculate values of the total inelasticity $Q$. Rather, these must be evaluated or assumed separately and supplied as input to the calculations. This model then evaluates the value of $\left(T_{R} / T_{R}^{o}\right)$ for selected values of laboratory-frame scattering angle $\theta_{\mathrm{R}}$ of the reactant ion, and for assumed values of $\left(Q / T_{R}^{\circ}\right)$. No assumptions are made concerning the disposition of $Q$ among internal modes of reactant ion and target, and emitted radiation (eq 2). The model is a simple consequence of the conservation of energy (eq 2) and of linear momentum in the two orthogonal directions defining the plane of the collision [23]. However, the momentum conservation equations, considered in this model, involve the precollision and postcollision momenta of the entire reactant ion (and also of the entire target gas molecule, in the event that this is not monatomic). These momentum conservation equations must, of course, be satisfied in any event, but it is the omission of details of all intermediate steps that results in $Q$ being undetermined by the model and its consequent appearance as an input parameter for the calculation. On the other hand, this disadvantage of the elastic-limit model is balanced by its flexibility in accounting for any and all contributions to $Q$, including electronic as well as rotational/vibrational excitation, or a combination thereof. The result of these considerations leads [23] to the following relationship:

$$
\begin{aligned}
{[1+} & \left.\left(m_{\mathrm{G}} / \mathrm{m}_{\mathrm{R}}\right)\right]^{2}\left(\mathrm{~T}_{\mathrm{R}} / \mathrm{T}_{\mathrm{R}}^{\mathrm{o}}\right) \\
= & \left\{\cos \theta_{\mathrm{R}} \pm\left[\cos ^{2} \theta_{\mathrm{R}}-\left(1-\mathrm{m}_{\mathrm{G}}^{2} / \mathrm{m}_{\mathrm{R}}^{2}\right)\right.\right. \\
& \left.\left.-\left(\mathrm{m}_{\mathrm{G}} / \mathrm{m}_{\mathrm{R}}\right)\left(1+\mathrm{m}_{\mathrm{G}} / \mathrm{m}_{\mathrm{R}}\right)\left(\mathrm{Q} / \mathrm{T}_{\mathrm{R}}^{\mathrm{o}}\right)\right]^{1 / 2}\right\}^{2}
\end{aligned}
$$

where all quantities are defined in a laboratory-fixed reference frame. Derivation of eq 3 also assumes [23] that $T_{G}^{o}$ is negligible, so that $\Delta T_{G}$ (eq 2) is approximately $T_{G}$, which is valid for the present example of 8 
$\mathrm{keV}$ ions colliding with thermal target atoms. The choice of sign for the second term on the right side of eq 3 corresponds to the "soft-hard collision" dichotomy discussed previously [23]. In the present work, the hard collisions (positive sign in eq 3 ) are not considered, since they are predicted to correspond to $\Delta T_{R R}$ values of up to several hundreds of electronvolts and are believed [37, 38] to have much lower cross sections than their soft counterparts.

In contrast to the elastic-limit model summarized by eq 3 , the so-called binary limit of the momentumtransfer model of CA considers a polyatomic reactant ion to be composed of two parts, an "impact" portion that interacts (exchanges momentum) with the target while the other is assumed to remain as a "spectator" for the duration of the collision. Such an interaction leads to rotational/vibrational excitation of the ion (but does not necessarily exclude simultaneous electronic excitation). Early applications [39-41] of this model to CID of simple ions (predominantly $\mathrm{H}_{2}^{+}$) included estimates of dissociation cross sections. However, the most common use of the binary-limit concept has involved comparison of the predicted values of $\Delta \mathrm{T}_{\mathrm{R}}$ with those predicted by elastic-limit calculations. Examples of such applications include scattering of $\mathrm{N}_{2}^{+}$on $\mathrm{Ar}$ [42] and of $\mathrm{Ar}^{+}$on $\mathrm{N}_{2}$ [43]. The mathematical expressions for $\Delta T_{R}$ as a function of $\theta_{R}$ have been converiently summarized [23]. More recently, Uggerud and Derrick et al. [44, 45] have succeeded in deriving expressions for $Q$ (as well as for $\Delta T_{R}$ ) within the binary limit, under the restriction that only vibrational/rotational excitation is considered. This represents a considerable advance, although there remains a requirement to assume how the reactant mass is to be divided between impact and spectator portions. Uggerud and Derrick et al. [44, 45] stated that their impact portions were assumed to be single constituent atoms of the reactant ion, but their treatment is more generally applicable.

Of the relationships thus deduced by Uggerud and Derrick et al. [44, 45], the most uscful for the present work are:

$$
\begin{gathered}
\Delta \mathrm{T}_{\mathrm{R}} / \mathrm{T}_{\mathrm{R}}^{\mathrm{u}}=\left(2 / \mu^{2}\right) \times\left\{\left(\mu-\cos ^{2} \theta_{\mathrm{R}}\right)\right. \\
\left. \pm\left[\left(\mu-\cos ^{2} \theta_{\mathrm{R}}\right)^{2}-\mu^{2}\left(1-\cos ^{2} \theta_{\mathrm{R}}\right)\right]^{1 / 2}\right\} \\
\mathrm{Q} / \Delta \mathrm{T}_{\mathrm{R}}=\mu / 2 \epsilon \\
\epsilon=\left[1+\left(\mathrm{m}_{\mathrm{a}} / \mathrm{m}_{\mathrm{G}}\right)\right] /\left\{2 \times\left[1-\left(\mathrm{m}_{\mathrm{a}} / \mathrm{m}_{\mathrm{R}}\right)\right]\right\} \\
\mu=\left[1+\left(\mathrm{m}_{\mathrm{a}} / \mathrm{m}_{\mathrm{G}}\right)\right] /\left[1+\left(\mathrm{m}_{\mathrm{a}} / \mathrm{m}_{\mathrm{G}}\right)-\left(\mathrm{m}_{\mathrm{a}} / \mathrm{m}_{\mathrm{R}}\right)\right]
\end{gathered}
$$

where $m_{R}$ and $m_{G}$ are written $a s m_{\text {ion }}$ and $m_{g}$, respectively, in the original work $[44,45]$, and $m_{a}$ denotes the mass of the impact portion (originally assumed [44] to be a single atom) of the reactant ion. Equations
4,5 , and 7 correspond respectively to eqs 41,42 , and 39 of the original work [44], reexpressed for convenience in terms of dimensionless mass ratios, while eq 6 is the correct expression for $\epsilon$, given as eq 2 in the follow-up work [45], which corrected errors in the original [44]. In this model, $Q$ is determined as a fixed fraction of $\Delta T_{R}$ (eq 5) and is not an input parameter. The hard-soft collision dichotomy (see eq 3) appears also in eq 4.

It is important to emphasize a fundamental limitation of all relationships derived from only considerations of conservation of energy and momentum. No attempt is made in such derivations to specify the $\mathrm{R}^{+}-\mathrm{G}$ interaction potential, so that the equations of motion are not considered in any way and thus no information is available concerning relative cross sections for competing outcomes. For example, eq 3 permits, within the elastic-limit approximation, calculation of $\Delta \mathrm{T}_{\mathrm{R}}^{(1)}$ for $\mathrm{R}^{+}$scattered through angle $\theta_{\mathrm{R}}^{(1)}$ with inelasticity $Q^{(1)}$, and also of $\Delta T_{R}^{(2)}$ for $R^{+}$scattered through $\theta_{R}^{(2)}$ with inelasticity $Q^{(2)}$. However, such a calculation provides no information concerning the relative probabilities of these two events. The only caveat to this conclusion concerns cases in which the specifications of the collision are inconsistent with the conservation requirements, a condition signaled by a negative value for the expression within square brackets (in either eq 3 or eq 4) whose square root must be evaluated; in such cases, of course, the cross section is zern. The limiting case of eq 3 , in which this expression in square brackets is exactly zero and in addition $\theta_{\mathrm{R}}$ is zero (head-on collision), corresponds to the conventional calculation of the center-of-mass collision energy $\left(\mathrm{T}_{\mathrm{COM}}\right)$, the maximum translational energy available for conversion into internal energy:

$$
\mathrm{Q}_{\max } / \mathrm{T}_{\mathrm{R}}^{\mathrm{o}}=\mathrm{T}_{\mathrm{COM}} / \mathrm{T}_{\mathrm{R}}^{\mathrm{o}}=\mathrm{m}_{\mathrm{G}} /\left(\mathrm{m}_{\mathrm{R}}+\mathrm{m}_{\mathrm{G}}\right)
$$

Again, eq 8 contains no information concerning the probability of such an occurrence.

To compare the two models with respect to interpretation of the expcrimental data summarized in Figures $1-3$, it is necessary to estimate appropriate values of $Q$ for input into the elastic-limit model (eq 3). The ions of interest are $\mathrm{Cs}_{4} \mathrm{I}_{3}^{+}$ions that survived the collisions for the $25 \mu \mathrm{s}$ flight time through the energy analyzer to the detector. The dissociation channel of lowest critical energy, as judged from the unimolecular fragment spectrum, is that forming $\mathrm{Cs}_{3} \mathrm{I}_{2}^{+}$by expulsion of a neutral CsI fragment. It is possible to estimate $\Delta \mathrm{E}_{0}$, the thermodynamic zero-point energy difference for this reaction, from theoretical calculations [46] of the binding energies (relative to ionic dissociation limits) of the two ions, together with experimental [47] and theoretical [48] values of the dissociation energy of CsI (again relative to ionic dissociation limits). The resulting value for $\Delta \mathrm{E}_{0}$ of $1.46 \mathrm{eV}$ is subject to an uncertainty of some $\pm 0.02 \mathrm{eV}$ arising from errors in reading values from Figure 6 of ref 46. This thermodynamic quantity for the reaction is related to the kinetic 
parameter $Q_{C I D}$, the magnitude of internal energy deposited in $\mathrm{R}^{+}$by CA events leading to dissociation on the experimental time scale, via eq 9:

$$
\mathrm{Q}_{\mathrm{CID}}=\Delta \mathrm{E}_{\mathrm{o}}+\mathrm{T}+\epsilon_{\mathrm{P}}-\epsilon_{\mathrm{R}}^{\mathrm{o}}
$$

where $T$ is the kinetic-energy release value for the dissociation and $\epsilon_{\mathrm{P}}$ is the combined internal energy of the dissociation products. All quantities in eq 9, with the exception of $\Delta \mathrm{E}_{\mathrm{o}}$, are understood to represent mean values. The value of $\mathrm{T}$ for the reaction of interest has been measured $[49,50]$ as $0.17 \mathrm{eV}$. The internal energies of the dissociation products are controlled by the detailed dynamics of the unimolecular fragmentation, and no quantitative information concerning $\epsilon_{\mathbf{P}}$ is available. The values of $\epsilon_{\mathrm{R}}^{\circ}$ are controlled by energy deposition during the sputtering process and by preferential depletion of ions with higher values of $\epsilon_{R}^{\circ}$ due to dissociation during flight between the ion source and the collision cell. In view of the intense metastable peak observed for this reaction, the population of reactant ions must include some with values of $\epsilon_{R}^{0} \geq$ $\left(\Delta E_{0}+T\right)$, corresponding to values of $Q_{C I D}(e q 9)$ close to zero. At the other extreme, the sputtering process presumably produces some ions with $\epsilon_{\mathrm{R}}^{\mathrm{o}}$ close to zero, and such ions will requirc (eq 9) $Q_{\mathrm{CID}}-(1.46+0.17$ $\left.+\epsilon_{\mathrm{p}}\right) \mathrm{eV}$ to lead to observable dissociation. The present experiments are concerned with survivor ions that do not undergo $C I D$ (i.e., ions with $Q<Q_{C I D}$ ). In view of these energetic considerations, the elastic-limit cal- culations were conducted for $Q$ values of $1 \mathrm{eV}$ and 3 $\mathrm{eV}$ to ensure that the relevant range was covered.

The relevant range of values for $\epsilon_{R}$ (and thus $Q$ ) is defined by the physical dimensions of the apparatus via the corresponding time windows and dissociation rate constants, and in particular is independent of the nature of the collision gas. It is thus pertinent to compare values of $\Delta T_{R}$ calculated for different targets $G$, at the same angle $\theta_{R}$ and for the same value of $Q$. The results of such calculations based on the elasticlimit model (eq 3) are shown in Figure 4. The difference between $\Delta T_{R}$ and $Q$ corresponds (eq 2) to $\Delta T_{G}$ when $\epsilon_{\mathrm{G}}$ is zero, and is much larger for helium than for neon. For yet heavier targets, the predicted difference between $\Delta T_{R}$ and $Q$ is even smaller than for neon, but these calculations have been omitted from Figure 4 in the interests of clarity. The physical reason underlying this consequence of eq 3 is simply the relationship between translational energy $\left(\alpha v^{2}\right)$ and momentum $\left(\propto v^{1}\right)$, where $v$ is the magnitude of the velocity. For two collision gases $\mathrm{G} 1$ and $\mathrm{G} 2$ at fixed values of $Q$ and of $\theta_{\mathrm{R}}$, a small ratio $\left(\mathrm{m}_{\mathrm{G} 1} / \mathrm{m}_{\mathrm{G} 2}\right)$ requires a correspondingly large ratio $\left(v_{\mathrm{G} 1} / \mathrm{v}_{\mathrm{G} 2}\right)$ for momentum conservation to be satisfied, and this implies an even larger difference when the $\mathrm{v}^{2}$ ratio is considered.

It is also possible to interpret Figure 4 in a fashion directly related to the experimental peak widths exemplified in Figures 1-3. The experimental uncertainty in $\theta_{R}\left( \pm 0.03^{\circ}\right)$ implies that a corresponding range of values of $\Delta T_{R}$ should be considered. For example, for

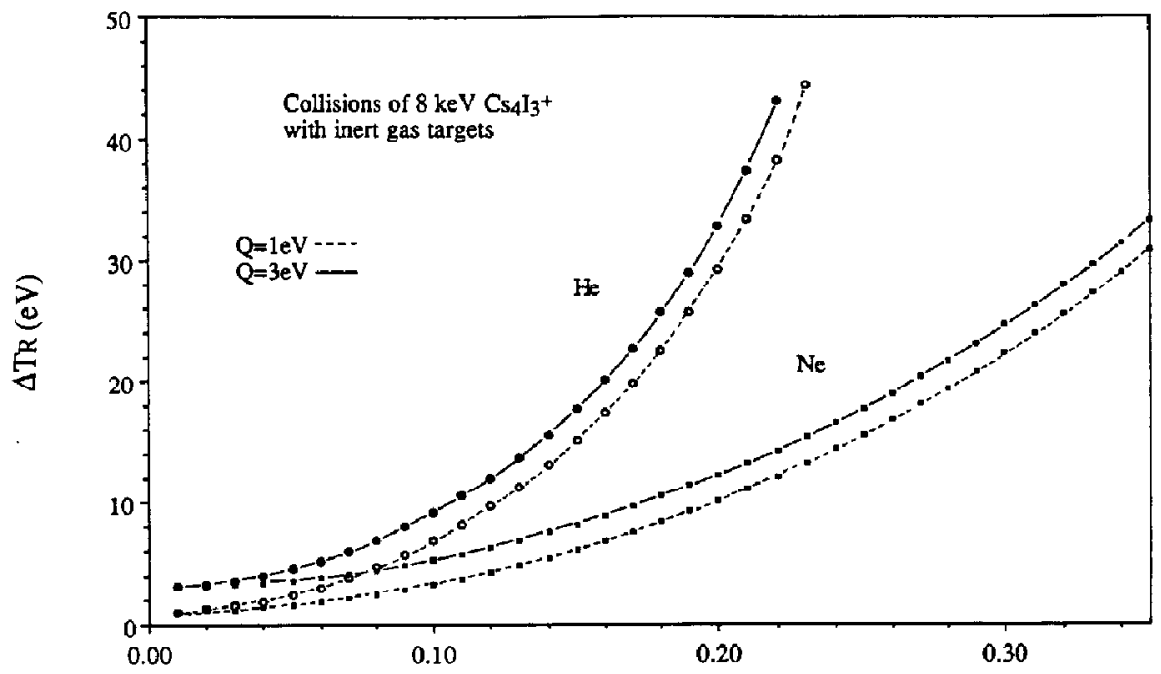

Angle (Deg.)

Figure 4. Values of translational energy loss $\Delta T_{R}$, calculated using the elastic-limit model (eq 3), for reactant ions $R^{+}$of mass $912 \mathrm{Da}$ and translational energy $8 \mathrm{keV}$ in collision with thermal target atoms $\mathrm{G}$ of mass $4 \mathrm{Da}(\mathrm{He})$ or $20 \mathrm{Da}(\mathrm{Ne})$, as a function of scattering angle $\theta_{\mathrm{R}}$. Representative values of the inelasticity $Q$ were estimated (see text) to yield dissociation rate constants sufficiently small $\left(\leq 10^{5} s^{-1}\right)$ that the activated ions would survive the $25 \mu$ s flight time from collision cell to detector. In the present experiments, $\theta_{\mathrm{R}}$ could be specified to within $\pm 0.03^{\circ}$, and $\Delta \mathrm{T}_{\mathrm{R}}$ to within $\pm 2 \mathrm{eV}$. 
an experimentally defined angle $\theta_{\mathrm{R}}$ of $0.15 \pm 0.03^{\circ}$, and for $Q$ in the range $1-3 \mathrm{eV}$, Figure 4 predicts ranges for $\Delta T_{R}$ of $10-26 \mathrm{eV}$ and $4-10.5 \mathrm{eV}$ for $\mathrm{He}$ and $\mathrm{Ne}$, respectively, as collision gases for $\mathrm{Cs}_{4} \mathrm{I}_{3}^{+}$at $8 \mathrm{keV}$. These calculated ranges correspond closely to those defined by the experimental peaks in Figures $1 \mathrm{~b}$ and $2 \mathrm{~b}$, measured at $25 \%$ of peak height. This degree of quantifative agreement between experiment and elastic-limit model calculations (eq 3) should probably be regarded as fortuitous; however, it is reassuring that the experimental trends are reproduced by the theory.

Calculations based upon the Uggerud-Derrick theory (eqs $4-7$ ) are summarized in Figure 5. In this case, $Q$ is calculated as a result of the theory rather than treated as an adjustable parameter. However, it is necessary to choose a value for $\mathrm{m}_{\alpha}$, the mass of the "impact portion" of the reactant ion. For the present calculations, $\mathrm{m}_{\mathrm{a}}$ was chosen to be $130 \mathrm{Da}$, representative of the atomic masses of $\mathrm{Cs}$ and I. The marked separation between the curve for helium and those for all other rare gas targets is still evident (Figure 5), but now there is a greater distinction among the curves for all collision gases other than helium. Real-valued solutions to eq 4 do not exist for $\theta_{\mathrm{R}}>\arcsin (\mu-1)$, reflecting the impossibility of conserving momentum for larger scattering angles when the discrepancy between

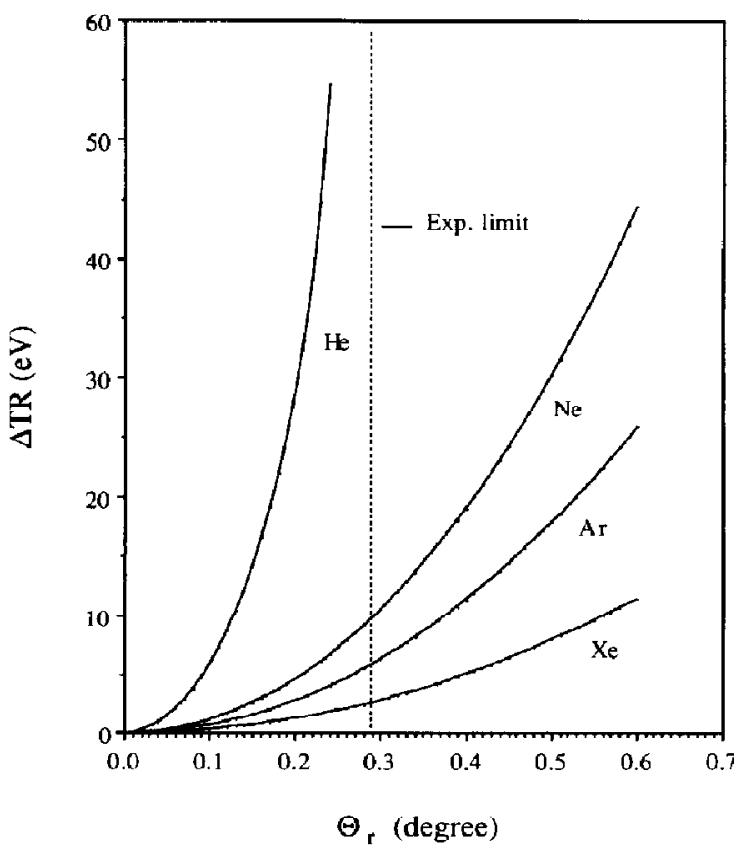

Figure 5. Values of translational energy loss $\Delta T_{R}$, calculated using the theory of Uggerud and Derrick et al. [44, 45] (eqs 4-7), for reactant ions $R^{\prime}$ of mass $912 \mathrm{Da}$ and translational energy 8 $\mathrm{keV}$ in collision with inert gas targets, as a function of scattering angle $\theta_{R}$. The value of $m_{a}$, the impact portion of the projectile ion, was taken to be $130 \mathrm{Da}$. The values of $Q / \Delta T_{R}$ calculated from eq 5 are: $\mathrm{He}, 0.026 ; \mathrm{Ne}, 0.127 ; \mathrm{Ar}, 0.209$; Xe, 0.462 . masses of the ion and the target is too large. For $m_{a}=130 \mathrm{Da}$ and $m_{R}=912 \mathrm{Da}$, these limiting values for $\theta_{R}$ are $0.245^{\circ}$ for helium and $1.21^{\circ}$ for neon, relative to the instrumental limit of $0.29^{\circ}$. In this regard, note the extremely weak signals observed for $\mathrm{He}$ at $0.26 \pm$ $0.03^{\circ}$ (very close to the limit) in Figure 1c, compared with those for Ne in Figure 2c. Thus, according to the Uggerud-Derrick model with $m_{a}$ chosen to be $130 \mathrm{Da}$, the maximum energy that can be deposited in an 8 $\mathrm{keV} \mathrm{Cs} \mathrm{I}_{3}^{+}$ion via collision with helium corresponds to this limit, evaluated as $1.8 \mathrm{eV}$ at a laboratory scattering angle of $0.245^{\circ}$. At the experimental limit for $\theta_{R}$ of $0.29^{\circ}$, the theory predicts values of $Q$ of $1.3 \mathrm{eV}$ for each of $\mathrm{Ne}, \mathrm{Ar}$, and $\mathrm{Xe}$. These values for $\mathrm{Q}$ lie well within the range estimated above based upon independent information on the energetics of $\mathrm{Cs}_{\mathrm{n}+1} \mathrm{I}_{\mathrm{n}}^{+}$ions, and are therefore consistent with the present observations (Figures 1-3) that $\mathrm{Cs}_{4} \mathrm{I}_{3}^{+}$can be scattered through such angles and survive for $25 \mu \mathrm{s}$ without dissociation.

Alternative proposals [13] that attribute variations in $\Delta \mathrm{T}_{\mathrm{R}}$ to obligatory excitation of the different targets cannot be tested by the theoretical models employed here. The most relevant experimental cvidence involves the indistinguishable data obtained (Figure 3) for $\mathrm{D}_{2}$ and for $\mathrm{He}$ as collision gases. The excitation and ionization energies of these two gases are very different (Table 1), which presents a serious problem for the interpretation [13] in terms of target excitation as the major sink for $\Delta \mathrm{T}_{\mathrm{R}}$. Further, the great majority of the present experimental data (Figure 3) for inert gas targets correspond to measured values of $\Delta T_{R}$ that are less than the lowest excitation energies (Table 1). The present interpretation in terms of target translational energy $T_{G}$ can accommodate both of these facts, although the helium-deuterium comparison does face a minor ambiguity in that it involves a monatomic and a diatomic collision gas. It would clearly be preferable to have a direct experimental test that could detect large translational energies for the scattered target gas atoms.

It does not seem possible to devise an experiment to detect such fast atoms in apparatus of the kind used in the present work. A crossed-beam experiment, employing coincident detection of scattered ions and target atoms, could in principle provide such evidence, and such experiments have been reported [51] for atomic ions scattered off atomic targets. The closest experimental analogy to the present work appears to be that of Morgan et al. [36], who measured excess translational energies (analogous to $\Delta \mathrm{T}_{\mathrm{G}}$ ) of target gas ions formed in $4 \mathrm{keV}$ collisions with neutral projectiles. The cross sections for these ionizing collisions were invariably low for neutral inert gas projectiles, and were zero for organic projectiles with helium as the collision target [36]. On the other hand, these workers [36] found the excess translational energies of the ionized target gas to be large (up to $10 \mathrm{eV}$ ) for heavy projectiles (e.g., $\mathrm{Xe}^{\mathrm{o}}$ ) in collision with light targets (e.g., $\mathrm{H}_{2}$ ), but these excess energies decreased to much smaller values as the mass ratio approached unity. The 
simple elastic-limit collision theory (eq 3) was invoked to interpret these data, although not all aspects of the phenomenon could be accounted for.

Increasing values of $\Delta T_{R}$ (most probable values measured at peak maximum) and an increasing extent of the low-energy tails of MIKES peaks for CA survivor ions, as the mass of the target is decreased, were observed $[18,19]$ in experiments in which beam collimation was not applied. lihese observations reflect the theoretical requirement that the range of $\Delta T_{R}$ values corresponding to the instrument-defined range for $\theta_{\mathrm{R}}$ is much larger in the case of a low-mass target (Figures 3 and 5). Thus, integration over $\theta_{R}$ implies superposition of $R^{+}$survivor ions with a wider range of $\Delta T_{R}$ values in the case of the low-mass targets, accounting qualitatively for these observations. Because nothing is known about the $\mathrm{R}^{+}-\mathrm{G}$ interaction potential, no predictions can be made concerning the relative probabilities for the various $\left(\Delta T_{R}, \theta_{R}\right)$ combinations, and thus no detailed quantitative interpretations of the peak shapes can be made using either of the theoretical approaches employed here.

\section{Conclusions}

The present experimental data on variation of translational energy spectra of CA survivor ions, with scattering angle and with nature of the collision gas, have been shown to be consistent with simple theoretical expressions derived from considerations of conservation of energy and linear momentum. The elastic-limit theory makes no mechanistic assumptions but requires that the total inelasticity $Q$ be estimated independently as an adjustable parameter. The particular expression of "binary-model" collision theory, derived by Uggerud and Derrick et al. [44, 45], assumes that electronic excitation is not involved and calculates $Q$ as a defined fraction of $\Delta T_{R}$. Both of these theoretical approaches can account qualitatively and semiquantitatively for all of the present observations, and both interpret the difference $\left(\Delta T_{R}-Q\right)$ as $T_{G}$, the recoil energy of the target. The alternative interpretation proposed by Bricker and Russell [13], in which this difference is attributed to obligatory excitation (or possibly ionization) of the target, is not applicable to the majority of the present observations for which the values of $\Delta T_{k}$ were chosen to be less than the lowest excitation energies of the appropriate rare gas targets. Further, this proposal [13] cannot account for the observation that use of helium and deuterium yiclded almost indistinguishable results, which differed markedly from those obtained using all other collision gases. Although the present results strongly support the recoil-energy interpretation, some ambiguities remain. For example, only one reactant ion was examined in this work; it was not possible to find a large orgaric ion at sufficient intensity that beam collimation was feasible. Further, the possibility that internal energy of the reactant ion provides a major sink for $\Delta T_{R}$ has not been ruled out definitively by the present experiments alone. A complementary approach, which attempts to assess the internal energies of the survivor ions, is described in an accompanying article [52].

\section{References}

1. Durup, J. In: Recent Developments in Mass Spectrometry; Ogata, K.; Hayakawa, T., Eds.; University of Tokyo Press: Tokyo, 1970; pp 921-934.

2. Los, J.; Govers, T. R. In: Colliston Spectroscopy; Cooks. R. G., Ed.; Plenum: New York, 1978; pp 289-356.

3. Shukla, A. K.; Qian, K.; Howard, S. L.; Anderson, S. G.; Sohlberg, K. W.; Futrell, J. H. Int. J. Mass Spectrom. Ion Processes 1989, 92, 147-169.

4. Qian, K.; Shukla, A. K.; Howard, S. L.; Anderson, S. G.; Futrell, J. H. J. Phys. Chem. 1989, 93, 3889-3892.

5. Shuklu, A. K.; Qian, K.; Anderson, S. G.; Futrell, J. H. J. Am. Soc. Mass Spectrom. 1990, 1, 6-15.

6. Singh, S.; Harris, F. M.; Boyd, R. K.; Beynon, J. H. Int. J. Mass Spectrom. Ion Processes 1985, 66, 131-149, 151-166.

7. DeKrey, M. J.; Kenttamaa, H. I; Wysocki, V. H.; Cooks, R. G. Org. Mass Spectrom. 1986, 21, 193-195.

8. Vincenti, M.; Horning, S. R.; Cooks, R. G. Org. Mass Spectrom. $1988,23,585-593$.

9. Cooks, R. G.; Ast, T.; Kralj, B.; Kramer, V.; Zigon, D. J. Am. Soc. Mass Spectrom. 1990, 1, 16-27.

10. McLuckey, S. A. J. Am. Soc. Mass Spectrom. 1992, 3, 599-614.

11. Neumann, G. M; Derrick, P. J. Org. Mass Spectrom. 1984, 19, 165-170.

12. Neumann, G. M.; Sheil, M. M.; Derrick, P. J. Z. Naturforsch. 1984, 39a, 584-592.

13. Bricker, D. L.; Russell, D. H. I. Am. Chem. Soc. 1986, 108, 6174-6179.

14. Guevremont, R.; Boyd, R. K. Rapid Commun. Mass Spectrom. 1988, 2, 1-5.

15. Sheil, M. M.; Derrick, P. J. Org. Mass Spectrom. 1988, 23, 429-435.

16. Uggerud, E.; Derrick, P. J. Z. Naturforsch. 1989, 44a, 245-246.

17. Alexander, A. J; Thibault, P.; Boyd, R. K. J. Am. Chem. Soc. $1990,112,2484-2491$.

18. Cerny, R. L.; Tomer, K. B.; Giblin, D. E. Proceedings of the 34th ASMS Conference on Mass Spectrometry and Allied Topics; Cincinnati, OH, 1986; pp 99-100.

19. Gross, M. L.; Tomer, K. B.; Cerny, R. L.; Giblin, D. E. In: Mass Spectrometry in the Analysis of Large Molecules; McNeal, C. J., Ed.; Wiley: New York, 1986; pp 171-190.

20. Alexander, A. J.; Thibault, P. Rapid Commun. Mass Spectrom. 1988, 2, 224-228.

21. Alexander, A. J.; Boyd, R. K.; Thibault, P.; Tomer, K. B. Proceedings of the 37th ASMS Conference on Mass Spectrometry and Allied Topics; Miami Beach, FL, 1989; pp 226-227.

22. Ballard, K. D.; Gaskell, S. J. I. Am. Soc. Mass Spectrom. 1992, 3, 644-655.

23. Boyd, R. K.; Kingston, E. E.; Brenton, A. G;; Beynon, J. H. Proc. Roy. Sac. Lond. 1984, A392, 59-88, 89-106.

24. Leventhal, J. J. In: Gas Phase Ion Chemistry, Vol. 3; Bowers, M. T., Ed.; Academic: New York, 1984; pp 309-355.

25. Maier, J. P. In: Kinetics of Ion-Molecule Reactions; Ausloos, P., Ed.; Plenum: New York, 1979; Pp 437-462.

26. Holmes, J. H.; Mayer, P. M.; Mommers, A. A. T. Am. Chem. Soc. 1991, 113, 9405-9406.

27. Holmes, J. H.; Mayer, P. M.; Mommers, A. A. Org. Mass Spectrom. 1992, 27, 537539. 
28. Holmes, J. H.; Mayer, P. M.; Mommers, A. A. Proceedings of the 40th ASMS Conference on Mass Spectrometry and Allied Topics; Washington, DC, 1992; pp 831-832.

29. Mommers, A. A. Personal communication.

30. Asamoto, B.; Dunbar, R. C. Chem. Phys. Lett. 1987, 139, 225-231.

31. Ahmed, M. S.; So, H. Y.; Dunbar, R. C. Chem. Phys. Lett. 1988, 151, 128-132.

32. Dunbar, R. C. J. Chem. Phys. 1989, 90, 7369-7375.

33. Huang, F.-S.; Durbar, R. C. J. Am. Chem. Soc. 1989, 111, 6497-6500.

34. Kim, M. S. Int. J. Mass Spectrom. Ion Phys. 1983, 50, 189-203.

35. Harrison, A. G.; Mercer, R. S.; Reiner. E. J.: Young. A. B.; Boyd, R. K.; March, R. E.; Porter, C. J. Int. J. Mass Spectrom. Ion Processes 1986, 74, 13-31.

36. Morgan, T. G.; Brenton, A. G.; March, R. E.; Harris, F. M.; Beynon, J. H. Int. I. Mass Spectrom. Ion Processes 1985, 64, 299-314.

37. Hasted, J. B. Physics of Atomic Collisions, 2nd ed; Elsevier: Amsterdam, 1972.

38. Massey, H. S. W.; Gilbody, H. B. Electronic and Ionic Impact Phenomena, Vol. 4; Clarendon: Oxford, 1974.

39. Gerasimenko, V. I.; Oksjuk, Yu. D. Sov. Phys. JETP 1965, 21, 333-336.
40. Green, T. A. Phys. Rev. A 1970, 1, 1416-1423.

41. McClure, G. W. Peek, J. M. Dissociation in Heavy Particle Collisions; Wiley: New York, 1972.

42. Fayeton, J.; Pernot, A.; Fournier, P.; Barat, M. J. Physique 1971, 32, 743-750.

43. Fernandez, S. M.; Friksen, F. J.; Bray, A. V; Pollack. E. Phys. Rev. A 1975, 12, 1252-1260.

44. Uggerud, E.; Derrick, P. J. J. Phys. Chem. 1991, 95, 1430-1436.

45. Cooper, H. J.; Derrick, P. J.; Jenkins, H. D. B.; Uggerud, E. I. Phys. Chem. 1993, 97, 5443-5444.

46. Diefenbach, J-; Martin, J. P. J. Chem. Phys, 1985, 83, 4585-4590.

47. Brumer, P.; Karplus, M. J. Chem. Phys. 1973, 58, 3903-3918.

48. Welch, D. O.; Lazareth, O. W.; Dienes, G. J.; Hatcher, R. D. I. Chem. Phys. 1976, 64, 835-839.

49. Hwang, H. J; Sensharma, D. K.; El-Sayed, M. A. J. Phys. Chem. 1989, 93, 5012-5015.

50. Hwang, H. J.; Sensharma, D. K.; El-Sayed, M. A. Chem. Phys. Lett. 1989, 160, 243-249.

51. Ewald, H.; Seibt, W. In: Recent Decelopments in Mass Spectrometry; Ogata, K.; Hayakawa, T., Eds.; University of Tokyo Press: Tokyo, 1970; pp 39-58.

52. Thibault, P.; Alexander, A. J.; Boyd, R. K.; Tomer, K. B. J. Am. Soc. Mass Spectrom. 1993, 4, 845-854. 